\title{
Maneuvers of Multi Perspective Media Retrieval
}

\author{
J. Hemavathy ${ }^{1}$, E. Arul Jothi ${ }^{2}$, R. Nishalini ${ }^{3 *}$, M. Oviya ${ }^{4}$ \\ ${ }^{1}$ Assistant Professor, Department of Information Technology, Panimalar Engineering College, Chennai, India \\ ${ }^{2,3,4}$ Student, Department of Information Technology, Panimalar Engineering College, Chennai, India \\ *Corresponding author: nishaliniraje@gmail.com
}

\begin{abstract}
Recently, Learning Machines have achieved a measure of success in the representation of multiple views. Since the effectiveness of data mining methods is highly dependent on the ability to produce data representation, learning multi-visual representation has become a very promising topic with widespread use. It is an emerging data mining guide that looks at multidisciplinary learning to improve overall performance. Multiview reading is also known as data integration or data integration from multiple feature sets. In general, learning the representation of multiple views is able to learn the informative and cohesive representation that leads to the improvement in the performance of predictors. Therefore, learning multi-view representation has been widely used in many real-world applications including media retrieval, native language processing, video analysis, and a recommendation program. We propose two main stages of learning multidisciplinary representation: (i) alignment of multidisciplinary representation, which aims to capture relationships between different perspectives on content alignment; (ii) a combination of different visual representations, which seeks to combine different aspects learned from many different perspectives into a single integrated representation. Both of these strategies seek to use the relevant information contained in most views to represent the data as a whole. In this project we use the concept of canonical integration analysis to get more details. Encouraged by the success of in-depth reading, in-depth reading representation of multiple theories has attracted a lot of attention in media access due to its ability to read explicit visual representation.
\end{abstract}

Keywords: Construction, Image input.

\section{Introduction}

Multi-visual representation learning faces the problem of multi-view data presentations that facilitate the release of information that is easy to use when making prediction types, data from a different view usually contains information that is relevant to the study of the representation of many views taking advantage of this point to read more complete presentations than those of the single view methods. Learning to represent multiple views has become a very promising topic with extensive use. The analysis of Canonical integration (CCA) and its kernel extensions with representation strategies in early studies for multidisciplinary representation learning. While the CCA and its kernel versions demonstrate their ability to model effectively in relationships between two or more dynamic sets, they are limited in finding high-quality organizations among multiple viewing data. Learning visual representation is a state of reading representation by narrating the details of multiple viewing data to enhance learning performance. Reading status cannot be matched to multi-view data structures. The representation obtained can also reduce learning performance. Methods for aligning multiple view representations seek to create alignment between presentations learned from different perspectives. Independent examples can be investigated from two factors namely alignment based on merger and distance and alignment based on similarity. Multiple visual representation learning strategies with alignment observations based on multiple combinations: canonical correlation analysis (CCA), junior CCA, kernel CCA, and in-depth CCA. Multi-view representation fusion methods aim to integrate multiple view input into a single integrated presentation. Independent examples can be reviewed in two ways.

\section{Literature Survey}

\section{A. Visual presentations thought of as multi-model embedding}

Authors: Guillem Collell, Ted Zhang, and Marie-Francine Moens

Explanation: Language and perspective provide relevant information. Combining both approaches to a single multimodal representation is an unresolved issue with a wide range of applications in the use of natural language and computer perspective. In this paper, we present a simple and effective method that learns the mapping of a language to the point of view and uses its visual prediction prediction to build multiple presentations. In this sense, our approach provides a well-thought-out way to construct presentations, consistent with the natural environment for reconstructing and integrating human memory. Using seven-dimensional conceptual simulation experiments shows that selected (or considered) vectors not only help to mix multimodal data, but also improve powerful non-model foundations and multidisciplinary technologies, thus demonstrating human-like judgment. Finally, this enlightening work enlightens us with the fundamental questions of natural language comprehension in relation to vision and language as the manifestations of many 
forms of integration and reconstruction.

B. Creating end-to-end interview programs using hierarchical neural network models

Authors: Iulian V. Serban, Alessandro Sordoni, Joshua Bengio, Aaron Courville, Joelle Pineau

Explanation: We are investigating the process of building an open, negotiated chat systems based on a large chat company using production models. Productive models generate system responses that are generated independently word-for-word, which opens up the possibility of practical and flexible interaction. To support this goal, we are expanding the newly proposed hierarchical encoder-decoder-decoder network in the chat space, and demonstrate that this model competes with state-of-the-art language models with backoff n-gram. It explores the limitations of these and other similar methods, and shows how its effectiveness can be improved by incorporating bootstrapping learning from large question answer pair corpus and embedding fake words.

\section{Cross-modal restoration with cnn visual cues: a new foundation}

Authors: Yunchao Wei, Yao Zhao, Lu Canyi, Shikui Wei

Explanation: Recently, visual features of the convolutional neural network $(\mathrm{CNN})$ have demonstrated their powerful potential as a universal manifestation of various recognition functions. In this paper, mod-modal retrieval with CNN visual features is done in a number of classic ways. Specifically, CNN visual features on the shelves are extracted from the CNN model, which is embedded in ImageNet with more than one million images from 1000 object categories, as a standard image for dealing with horizontal retrieval. Continuing to maximize the ability to represent CNN's visual features, according to the $\mathrm{CNN}$ model previously developed in ImageNet, a well-organized step is made using Caffe CNN's open source library for each targeted data. In addition, we propose a more consistent approach to addressing the problem of retrieval modems in relation to samples defined by one or more labels. A detailed examination of the publicly available data sets clearly shows the height of CNN's visual features for the opposite retrieval.

\section{Description of many videos}

Authors: Vasili Ramanishka, Abir Das, Dong Huk Park, Subhashini Venugopalan

Explanation: Real-world web videos often contain clues to add visual details to create natural language descriptions. In this paper we propose a sequential model that examines such assistive details. Specifically, audio and video title are used in addition to the visual information in the multi-channel framework to produce consistent descriptions of "wild" videos. In contrast to current embedding coding models that use only visual data during the coding phase, our model incorporates multiple sources of information intelligently, showing improvement rather than using a variety of different methods.
We have supported our multi-video definition network in stateof-the-art video-to-text (S2VT) sequence model, expanded it for the use multiple methods. Extensive testing of MSR-VTT challenging datasets was performed to demonstrate the high performance of the proposed method for natural videos available on the web.

\section{E. A reading of various presentations with random movement} on the click-through graph

Authors: Fei Wu, Xinyan Lu, Jun Song, Shuicheng Yan

Explanation: In the acquisition of multimedia information, many traditional methods often represent different media modes in the same feature space. For click-through data collected in user search, existing methods take one-to-one-pair data (text-image pairs) and measurement examples (text queryimage and/or image-query-text ranking list) as training examples, do not make full use of click-through data, in particular open communication between data objects. In this paper, we treat click-through data as a large click-through graph, in which the vertices are graphic/text queries and edges that show clicks between image and query. We look at learning multimodal representation with a view to encoding in a clear / obvious relationship between the vertices on the click graph. By reducing both the random loss of random movement and the distance between the learned representation of the vertices and their corresponding deep network output, the proposed model called the Multimodal Random Walk Neural Network (MRW$\mathrm{NN}$ ) can be used not only to read the firm representation of existing multimodal data on the click graph, but also invisible and images to support the opposite retrieval. We examine the hidden representations learned by MRW-NN in the large public click-to-click clickture data and continue to demonstrate that MRW-NN achieves the best performance of street access to questionable images/images than other state-of-the-art alternatives.

\section{Existing System}

In the present system, information can also be accessed in the form of texts or pictures. The user provides a query in search engines, and then writes text, links and images with the same keywords/tags or annotations, the idea of extracting information from an image that contains text, and then separating text into words and retrieving information based on search text. Users may find it difficult to use text to query a specific part of the image or video content. Text-based retrieval methods are strictly limited to requiring metadata tagged on an image or video. If the requested text is not marked with the same tag as attached to the photo or video, the data is not returned. Another disadvantage of text-based retrieval is that the word may have different meanings. This problem is best illustrated by the example of jaguar or Apple photos or videos.

\section{Proposed System}

The proposed system uses visual similarity (low-level 
features such as color, shape, texture) to retrieve images. The content of the image is used to search instead of text. These systems depend entirely on the content of the image. No keywords required for search. The image is analyzed; Features are collected and stored to retrieve similar images. This creates a unique and compact digital signature or fingerprint of the image and is compatible with other index images. The system serves as a search tool for the blind and a translator for people who do not know the language. Several important applications of the practice of multi-view representation are discussed. A large number of multi-view embedding methods have been proposed to address these challenges, including multi-view embedding modeling, scalable processing requirements and targets that are inappropriate for the existence of disagreement. Since the performance of machine learning methods relies heavily on the expressive power of data representation, the practice of multi-view representation has become very promising with wide application.

\section{Module}

1. Authentication module

2. Authorization module

3. Admin module

4. User module

5. Module of projects

6. Design module

7. Products and services module

8. Resources module

\section{A. Authentication model}

Authentication is the process of determining who or what, in fact, is who or what it declares itself to be.

\section{B. Authorization module}

Empowerment is the process of allowing someone to do or do anything. In multi-user computer systems, the system administrator defines what system privileges (file directory, access time, allocated storage space, and so on) are for the system that allows users to access the system.

\section{Admin module}

The administrator can upload all the details of the user that they have access to view the details. They handle all data in a highly secure and instant feedback to end users. They must check the customer who requested the product.

\section{User module}

Modules provide user details. If the user is new to the app, they must register the application. User can enter details with proper verification and all fields are required for the registration process.

\section{E. Module of projects}

The module provides complete and ongoing projects of the website. The user can also view completed and completed projects. They can see all the project details like architecture, builder, cost etc. Customers can study about the project and they can contact the builder if needed.

\section{F. Design Module}

The module provides details about the structure of a particular building. The user took a picture of the building and uploaded it to the search engine. If the required details are available, it shows the entire building, cost, style and interior architecture. Builder. Details are provided in image, audio, video format. If the image is blurry, the image is divided into seven sections and compared between the seven sections.

\section{G. Products and service modules}

The module provides products and services to the customer. The administrator uploads product and service details. Customers can view details of available products and services and purchase the products they need. They may also use the Services. The consumer can buy products like brick, sand, steel rod.

\section{H. Resources module}

The module builder provides the details associated with the specific website. Customers can book the required builder agencies through the website for their convenience. The administrator acts as an intermediary between the customer and the builder agency.

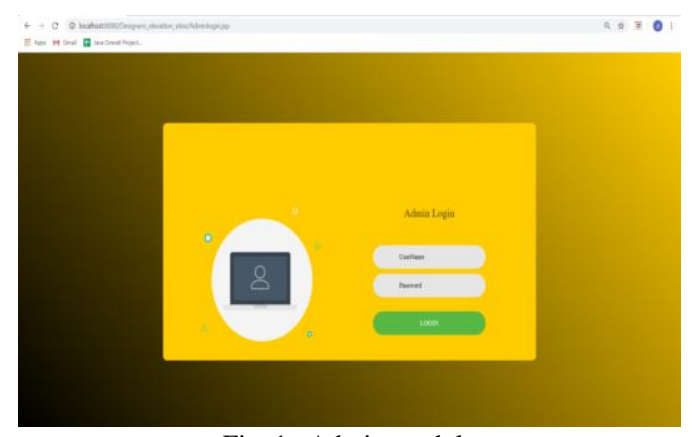

Fig. 1. Admin module

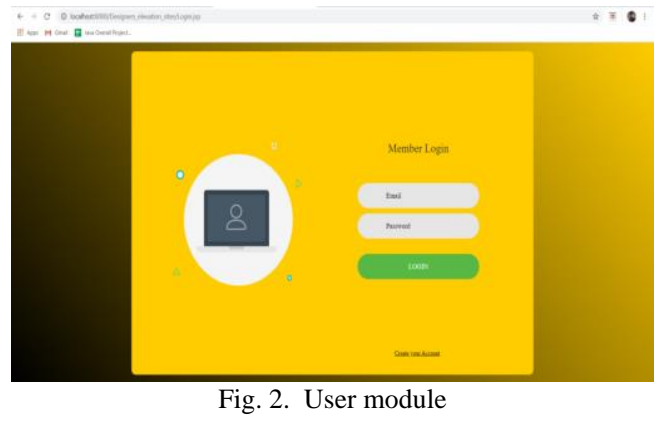

\section{Future Enhancements}

We plan to apply the material for a construction process such as natural language. We can apply this concept in the coming days, working without any framework support, which will reduce some time and business logic. They can be map 
configurations, so it's easy to run this process on multiple servers.

\section{Conclusion}

Multi-view representation learning is related to the problem of representation learning of multi-view data, which allows for the easy retrieval of useful information through canonical correlation analysis, which effectively correlates between two or more sets of variables. More popular for its efficiency. Multiview learning can also be called as data fusion or data integration from multiple feature sets. The purpose of this survey is to provide a practical picture of the theoretical foundations and current developments in the field of multivisual representation learning and to assist in finding an appropriate approach to specific applications. Our project will be to research future improvements on the exclusion that occurs on non-linearity, where when blur image is given as input, it does not give accurate results.

\section{References}

[1] E. Bruni, N. Kevin. Kevin. Tran, And M. Baroni, "Multimodal Distributive semantics," J. Artif. Intel. Race. (Zaire), Vol., No. 1-47, 2014.

[2] G. Collell, T. "Imaginary visual representation as multimodal embedding as ppm 4378-4384" in Aong, and M. Mons, AAAI, 2017.

[3] D. Fort et al. Botau, "Improvements in learning image using convolutional neural networks for multi-model semantics." EMNLP, 2014, pp. 36-45.

[4] A. M. Rush, S. Chopra, and J. Weston, "A Neural Attention Model for Abstract Sentence Abbreviations," Archive Print Archive: 1509.007, 5, 2015.

[5] O. Vinyl and Q. Lee, "A Neural Conversation Model," ArchivePrint RXIV: 1507.05: 79, 2015.

[6] I. V. Cerban, A. Sordoni, Y. Benzio, A. C. Cortville, and J. Pinow, "Constructive End-to-End Dialog Systems Using Generator Hierarchical Neural Network Models." In AAAI, Vol. 16, 2016, pp. 3776-3784

[7] V. Ramanishka, A. Das, D. H. Park, S. Venugopalan, La Hendrix, Rohrab, and Kevin. Sayenko, "Multimodal video description," Multimedia Conference 2016, Proceedings of the ACM in 2014. 1092-1096. 